\title{
Plasma flow and plasma-wall transition in Hall thruster channel
}

\author{
M. Keidar ${ }^{\text {a) }}$ and I. D. Boyd \\ Department of Aerospace Engineering, University of Michigan, Ann Arbor, Michigan 48109 \\ I. I. Beilis \\ Electrical Discharge and Plasma Laboratory, Fleischman Faculty of Engineering, Tel Aviv University, \\ P. O. Box 39040, Tel Aviv 69978, Israel
}

(Received 27 August 2001; accepted 2 October 2001)

\begin{abstract}
In this paper a model of the quasineutral plasma and the transition between the plasma and the dielectric wall in a Hall thruster channel is developed. The plasma is considered using a two-dimensional hydrodynamic approximation while the sheath in front of the dielectric surface is considered to be one dimensional and collisionless. The dielectric wall effect is taken into account by introducing an effective coefficient of the secondary electron emission (SEE), $s$. In order to develop a self-consistent model, the boundary parameters at the sheath edge (ion velocity and electric field) are obtained from the two-dimensional plasma bulk model. In the considered condition, i.e., ion temperature much smaller than that of electrons and significant ion acceleration in the axial direction, the presheath scale length becomes comparable to the channel width so that the plasma channel becomes an effective presheath. It is found that the radial ion velocity component at the plasma-sheath interface varies along the thruster channel from about $0.5 C_{s}\left(C_{s}\right.$ is the Bohm velocity) near the anode up to the Bohm velocity near the exit plane dependent on the SEE coefficient. In addition, the secondary electron emission significantly affects the electron temperature distribution along the channel. For instance in the case of $s=0.95$, the electron temperature peaks at about $16 \mathrm{eV}$, while in the case of $s=0.8$ it peaks at about $30 \mathrm{eV}$. The predicted electron temperature is close to that measured experimentally. The model predictions of the dependence of the current-voltage characteristic of the $E \times B$ discharge on the SEE coefficient are found to be consistent with experiment. (C) 2001 American Institute of Physics. [DOI: 10.1063/1.1421370]
\end{abstract}

\section{INTRODUCTION}

A Hall thruster is currently one of the most advanced and efficient types of electrostatic propulsion devices for spacecraft. The Hall thruster can offer much higher thrust density than other types of stationary ion thrusters. This configuration is beneficial because the acceleration takes place in a quasineutral plasma and thus is not limited by space charge effects. State of the art Hall thrusters have an efficiency of about 50\% with specific impulses in the range of 1000-3000 s. ${ }^{1}$ The electrical discharge in the Hall thruster has an $E$ $\times B$ configuration where the external magnetic field is radial and perpendicular to the axial electric field, which accelerates the ions. Passing the electron current across a magnetic field leads to an electron closed drift or Hall drift. The original idea of ion acceleration in the quasineutral plasma was introduced in mid-1960s (see Refs. 2-5) and since then numerous experimental and theoretical investigations have been conducted. The main results of these studies were summarized in recent reviews. ${ }^{6,7}$ Generally two different types of Hall thruster were developed: a thruster with closed electron drift and extended acceleration zone, or stationary plasma thruster (SPT), and a thruster with short acceleration channel or thruster with anode layer (TAL). In this paper we will

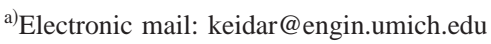

consider the first type of thruster, namely SPT, which employs a dielectric channel that plays an important role in the discharge.

In a SPT, the interaction of the plasma with the dielectric wall plays an important role. Due to the collisions of the electrons with the wall and secondary electron emission, the electron temperature remains relatively low in comparison to the TAL. As a result, the ion acceleration occurs over a more extended region. ${ }^{6,8}$ Despite many theoretical efforts, the complicated physical processes in the Hall thruster channel are far from being completely understood as was recognized in recent reports. ${ }^{1,6}$ Mainly the physics of the plasma interaction with a dielectric wall and the transition between quasineutral plasma and sheath have not been investigated in detail. However, it was shown experimentally that the dielectric material affects the discharge behavior in the Hall thruster. ${ }^{9}$ Very recently it was found that use of sectioned electrodes inside the Hall thruster channel have a considerable effect on the discharge characteristics as well as thruster performance. ${ }^{10,11}$ These findings suggest that the effect of the plasma interaction with a dielectric wall is an important issue in Hall thrusters. The transition plasma-wall region determines the particle and energy fluxes from the plasma to the wall. It is well known that, in the case when the wall has a negative potential with respect to the plasma, the stationary electrostatic sheath existence connects to the Bohm 
condition. ${ }^{12,13}$ Basically, this condition requires the ion acceleration up to an energy equal to that of the electrons in the quasineutral near-sheath region, called the presheath. The formulation of the presheath problem in a magnetic field was developed by Chodura ${ }^{14}$ in the collisionless limit and by Riemann ${ }^{15}$ in the weak collision case. In these models, the Boltzmann distribution (BD) for the electrons was used in order to determine the electric field. Generally, the electron distribution function may be described by a $\mathrm{BD}$ when the pressure gradient is equal to the electrical force. This will occur when the plasma flow is parallel to the magnetic field. ${ }^{16}$ For the case of plasma flow across a magnetic field, a fluid model was developed both for electrons and ions. ${ }^{17-19}$ It was shown that generally the electron distribution function deviates from the $\mathrm{BD}$. It was also shown that even when the plasma density dependence on the potential corresponds to the $\mathrm{BD}$, the presheath thickness deviates from that calculated with a model based on this assumption.

Basically two modeling approaches were undertaken in the past: particle simulation and hydrodynamic approach. The variation of the first approach is the hybrid models in which ions and neutrals are treated as particles whereas electrons are treated as a fluid. ${ }^{20-22}$ In this approach, however, very simplified boundary conditions are applied at the walls without considering the plasma-wall transition. Recently some attempt to include the near wall plasma effects was taken. ${ }^{23}$ In the second approach, the one-dimensional (1-D) hydrodynamic description for all species is employed. ${ }^{24-27}$ Generally it is assumed that the ion velocity at the quasineutral plasma edge is equal to the Bohm speed and this determines the ion losses to the wall. However, due to restrictions of 1-D analyses, the real boundary condition, e.g., presheath structure, where the conditions for the sheath entrance can be developed was not considered. Due to specific conditions of the plasma in Hall thrusters, i.e., ion temperature significantly smaller than electron temperature and substantial plasma acceleration in the axial direction, it is not clear how conditions for the sheath entrance are realized. In the present work we will specifically consider in detail the plasmasheath transition.

The present paper is organized as follows. In Sec. II we will present sheath-presheath interface analyses and the model of the sheath under the condition of strong secondary electron emission. Then the model of the quasineutral plasma (presheath) will be described followed by Sec. III, where the most important results are presented.

\section{PHYSICAL MODEL}

It will be shown in the following that the presheath scale length becomes comparable to the channel width under typical conditions of the Hall thruster plasma flow. Thus, the model for the quasineutral plasma region is extended up to the sheath edge in order to provide the boundary condition at the plasma-sheath interface as shown in Fig. 1. In the following sections, a model of the sheath in front of the dielectric wall, a quasineutral plasma presheath model, and conditions for a smooth transition between these regions are each described.

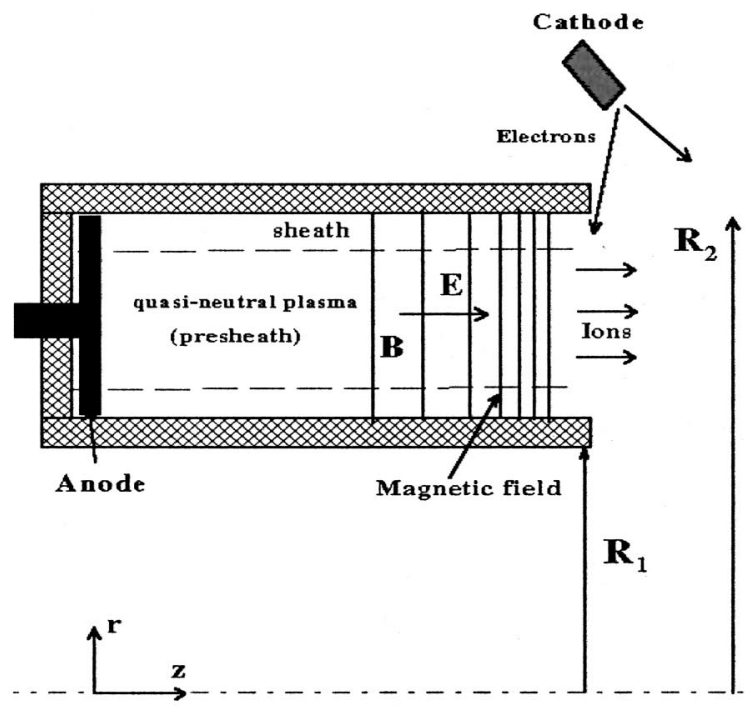

(a)

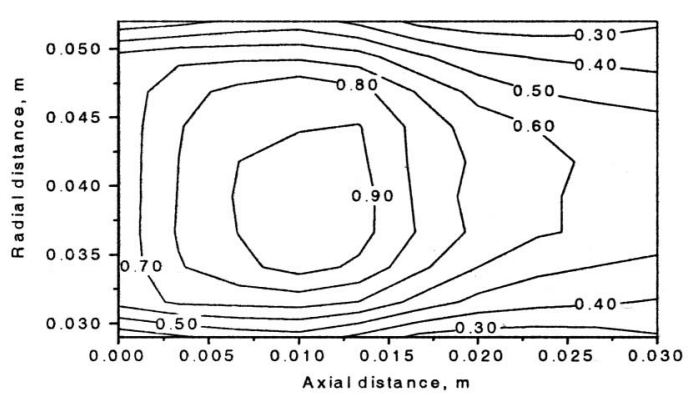

(b)

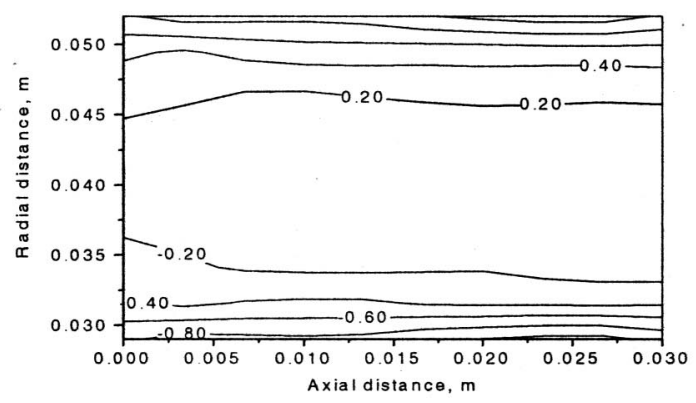

(c)

FIG. 1. (a) Schematic drawing of the acceleration channel of the Hall thruster with presheath and sheath regions (not to scale), (b) plasma density (normalized by the upstream neutral density), and (c) radial velocity component distributions (normalized by the Bohm velocity).

\section{A. Sheath and sheath-presheath interface}

The smooth transition between sheath and presheath is considered in the present work following the methodology developed previously by Beilis and Keidar. ${ }^{19}$ The necessary condition for the existence of a continuous solution for the sheath problem, when the electric field at the sheath edge is assumed to be equal to zero, is known as the Bohm criterion. However under this assumption, the electric field at the presheath edge approaches infinity (a singular point). In such a case, a smooth matching of the presheath and sheath solu- 


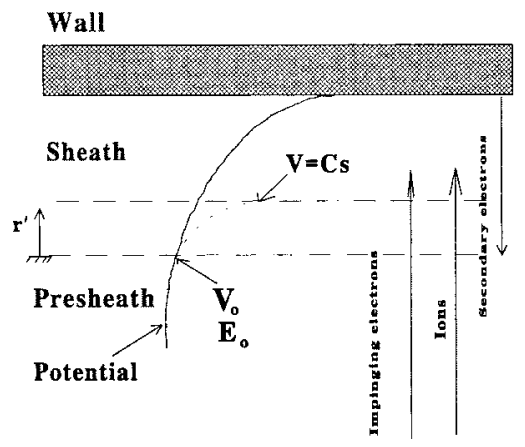

FIG. 2. Schematic of the plasma-wall transition layer. The boundary where the ion velocity is equal to the sound speed $\left(V=C_{s}\right)$ corresponds to the solution with infinite electric field at the presheath edge and zero electric field at the sheath edge. The boundary at $r^{\prime}=0$ corresponds to smooth transition with monotonic potential behavior across presheath and sheath.

tions is impossible. However, if the velocity at the sheath edge differs slightly from the Bohm velocity, the electric field becomes a continuous function as shown in Fig. 2. It was shown that a monotonic solution for the sheath problem could be obtained when the ion velocity at the sheath edge is smaller than the Bohm velocity. ${ }^{19,28,29}$ In this case, the electric field becomes a continuous function increasing from a small nonzero value at the sheath edge up to a maximum value at the wall as shown schematically in Fig. 2. The solution criterion in that case is the minimal velocity at the presheath-sheath interface that results in a continuous solution for the potential distribution in the sheath. ${ }^{19}$ The present work will take into account a nonzero electric field $E_{0}$ at the presheath-sheath interface that, in combination with the plasma velocity at that interface $V_{0}$, will determine the entrance conditions for the sheath.

Under typical steady state conditions, the potential drop between the plasma and the wall is negative in order to repel the excess of thermal electrons. However, when the wall has substantial secondary electron emission, the floating potential drop may be different from that in the simple sheath. This effect will be considered in this section.

In the considered range of parameters (see the following), the electrostatic sheath can be considered as collisionless and unmagnetized, since the Debye length is much less than the collision mean free path and the Larmor radius. We employ a one-dimensional sheath model that is based on the assumption that the sheath thickness is much smaller than the plasma channel width. Secondary electron emission from the dielectric wall is taken into account. For simplicity only singly charge ions are considered. The electrostatic potential distribution in the sheath satisfies the Poisson equation:

$$
\nabla^{2} \varphi=e / \varepsilon_{0}\left(n_{e 1}-n_{i}+n_{e 2}\right),
$$

where $\varphi$ is the potential, $n_{e 1}$ is the density of plasma electrons, $n_{e 2}$ is the density of secondary electrons, and $n_{i}$ is the ion density. The plasma electrons are assumed to obey the Boltzmann distribution:

$$
n_{e 1}=\left(n_{0}-n_{e 2}(0)\right) \exp \left(-e \varphi / k T_{e}\right),
$$

where $n_{e 2}(0)$ is the density of the secondary electrons at the sheath edge, $r^{\prime}=0$ (see Fig. 2), and $n_{0}$ is the electron density at the sheath edge that is equal to the ion density $n_{i}(0)$. The ions are assumed to be cold and have at the presheath-sheath interface the energy $U_{i 0}=1 / 2 m_{i} V_{0}^{2}$, where $V_{0}$ is the ion velocity at the sheath edge (see Fig. 2) and $m_{i}$ is the ion mass. Ions have free motion in the sheath and their density decreases according to

$$
n_{i}=n_{0}\left[1+2 e \varphi /\left(m V_{0}^{2}\right)\right]^{-0.5} .
$$

The current continuity equation can be written in the form:

$$
j_{e 1}+j_{i}-j_{e 2}=0
$$

where $j_{e 1}$ is the flux of the primary electrons from the plasma $\left(j=n V\right.$ is the flux definition for all species), $j_{i}$ is the ion flux, and $j_{e 2}$ is the flux of the secondary electrons. From this equation one can obtain that

$$
n_{e 2} V_{e 2}=s /(1-s) n_{0} V_{0},
$$

where $s$ is the secondary electron emission coefficient determined as $s=j_{e 2} / j_{e 1}$. We will furthermore assume that the electrons emitted from the surface are monoenergetic and freely move in the sheath. The boundary conditions for the sheath problem are the following:

$$
\varphi(0)=0, \quad d \varphi / d r\left(r^{\prime}=0\right)=E_{0}, \quad V=V_{0} .
$$

Two parameters are critical for the sheath solution: the electric field and the initial ion velocity. From the current continuity equation [Eq. (4)] one can calculate the potential drop across the sheath $\Delta \varphi_{w}$, as:

$$
\Delta \varphi_{w}=k T_{e} \ln \left((1-s) /\left[V_{0}\left(2 \pi m_{e} / k T_{e}\right)^{0.5}\right]\right) .
$$

This solution is similar to that described first by Hobbs and Wesson, ${ }^{30}$ except that they a priori assumed the Bohm velocity at the sheath edge. One can see that the potential drop across the sheath decreases with the secondary electron emission (SEE) coefficient. It should be noted that in this model we have assumed that a monotonic potential distribution exists. However, when the SEE coefficient $s$ approaches unity, the solution in form (7) breaks down and when $s$ exceeds a critical value, a potential well forms such that a fraction of emitted electrons are returned to the wall. This happens when $s$ is less than 1 and it was obtained that the critical value of the SEE coefficient $s^{*}$ can be calculated as ${ }^{30}$

$$
s^{*}=1-8.3\left(m_{e} / m_{i}\right)^{0.5} .
$$

From Eq. (8) it can be estimated that in the case of BN (the dielectric material usually used in SPT), the critical SEE coefficient is about 0.95 (Ref. 30). In the present work, only the monotonic potential distribution in the sheath will be considered.

\section{B. Plasma presheath model}

The presheath model is based on the assumption that the quasineutral region length (i.e., channel width $R_{2}-R_{1}$, see Fig. 1) is much larger than the Debye radius and therefore we will assume that $Z_{i} n_{i}=n_{e}=n$, where $Z_{i}$ is the ion mean charge, $n_{i}$ is the ion density, and $n_{e}$ is the electron density. For simplicity only single charge ions are considered in this paper $\left(Z_{i}=1\right)$. We will consider the plasma flow in a cylindrical channel as shown in Fig. 1. A magnetic field with only 
a radial component, $B_{r}=B$, is imposed. Cylindrical coordinates will be used, as shown in Fig. 1, with angle $\theta$, radius $r$, and axial distance from the anode $z$, respectively. The plasma flow starts in the near anode region and has lateral boundaries near the dielectric wall. The plasma presheath-sheath interface is considered to be the lateral boundary for the plasma flow region. A plasma will be considered with "magnetized" electrons and "unmagnetized" ions, i.e., $\rho_{e} \ll L$ $\ll \rho_{i}$, where $\rho_{e}$ and $\rho_{i}$ are the Larmor radii for the electrons and ions, respectively, and $L$ is the channel length. We employ a hydrodynamic model assuming: (i) the system reaches a steady state, (ii) electron temperature is consistent along $B$, and (iii) the electron component is not inertial, i.e., $\left(\mathbf{V}_{e} \boldsymbol{\nabla}\right) \cdot \mathbf{V}_{e}=0$. The following system of equations describes the quasineutral plasma:

$$
\begin{aligned}
& n m_{i}\left(\mathbf{V}_{i} \boldsymbol{\nabla}\right) \mathbf{V}_{i}=n e \mathbf{E}-\nabla P_{i}-\beta n m_{i} n_{a} \mathbf{V}_{a}, \\
& \boldsymbol{\nabla} \cdot\left(\mathbf{V}_{i} n\right)=\beta n n_{a}, \\
& \boldsymbol{\nabla} \cdot\left(\mathbf{V}_{a} n_{a}\right)=-\beta n n_{a}, \\
& 0=-e n(\mathbf{E}+\mathbf{V} \times \mathbf{B})-\boldsymbol{\nabla} P_{e}-n \nu_{m} m_{e} \mathbf{V}_{e}, \\
& \frac{3}{2} \partial\left(j_{e} T_{e}\right) / \partial z=Q_{j}-Q_{w}-Q_{\text {ion }},
\end{aligned}
$$

where $n$ is the plasma density, $\beta$ is the ionization rate, $Q_{j}$ $=j_{e} E$ is the Joule heat, $E$ is the axial component of the electric field, $j_{e}$ is the electron current density, $Q_{w}$ $=\nu_{w} n\left(2 k T_{e}+(1-s) e \Delta \varphi_{w}\right)$ represents the wall losses, ${ }^{26} \nu_{w}$ is the frequency of electron collisions with walls, $Q_{\text {ion }}$ $=e n_{a} n U_{i} \beta\left(T_{e}\right)$ represents ionization losses, $U_{i}$ is the ionization potential (for xenon, $U_{i}=12.1 \mathrm{eV}$ ), and $\beta\left(T_{e}\right)$ is the ionization coefficient (we will use the same expression as in Ref. 25). The last term in Eq. (9) stands for an effective drag force due to nonelastic collisions similar to that used in Ref. 31. To simplify the problem without missing the major physical effects, we consider one-dimensional flow of the neutrals. The equations for the heavy particles (ions and neutrals) may be written in component form in cylindrical coordinates by taking into account that the ion temperature is much smaller than the electron temperature (that makes it possible to neglect the ion pressure term in the momentum conservation equation):

$$
\begin{aligned}
& \frac{\partial\left(n V_{z}\right)}{\partial z}+\frac{\partial\left(n V_{r}\right)}{\partial r}+\frac{n V_{r}}{r}=\beta n_{i} n_{a}, \\
& V_{z} \frac{\partial V_{z}}{\partial z}=-V_{r} \frac{\partial V_{z}}{\partial r}+\frac{e}{m_{i}} E_{z}-\beta V_{a} n_{a}, \\
& V_{z} \frac{\partial V_{r}}{\partial z}=-V_{r} \frac{\partial V_{r}}{\partial r}+\frac{e}{m_{i}} E_{r}, \\
& \frac{\partial\left(n_{a} V_{a}\right)}{\partial z}=-\beta n_{i} n_{a} .
\end{aligned}
$$

In this model the electron flow [Eq. (12)] will be considered separately along and across magnetic field lines. Due to the configuration of the magnetic field (i.e., only the radial magnetic field component is considered in the model as shown in Fig. 1), the electron transport is greater in the azimuthal direction $(E \times B$ drift) than in the axial direction (drift diffusion due to collisions). According to Eq. (12), the electron transport equation along the magnetic field can be written as a balance between pressure and electric forces assuming that the current component in the radial direction is zero. If we assume that the electron temperature is constant along each magnetic field line we obtain that

$$
\varphi-\frac{k T_{e}}{e} \ln n=\text { const. }
$$

The left-hand side of Eq. (18) is known as a thermalized potential. ${ }^{7}$ This equation makes it possible to reduce the twodimensional calculation of the electric field to a onedimensional problem. According to Eq. (18) the electric field in the radial direction $E_{r}$ [in Eq. (16)] is determined by the electron pressure gradient in this direction. Calculating the potential distribution along the channel centerline makes it possible to calculate the potential in the entire domain using Eq. (18) similarly to Refs. 20 and 22. For known total discharge current and ion current fraction one can calculate the electron current fraction from the current continuity condition. The equation describing the electron transport across the magnetic field can be obtained from Eq. (12) and reads:

$$
j_{e z}=e n \frac{\mu_{e}}{1+\left(\omega_{e} / \nu_{m}\right)^{2}}\left(E_{z}+\frac{\partial T_{e}}{\partial_{z}}+T_{e} \frac{\partial \ln n}{\partial z}\right),
$$

where $\nu_{m}=\nu_{e n}+\nu_{e w}+\nu_{B}$ is the effective electron collision frequency. In the following we will determine different components of the effective electron collision frequency.

\section{Electron collisions}

For typical conditions of the Hall thruster, the effect of Coulomb collisions appears to be negligibly small ${ }^{21}$ and will not be considered here. The total electron collision frequency considered in the present model consists of electron-neutral collisions, electron-wall collisions, and anomalous collisions (Bohm diffusion). The electron-neutral collision frequency may be estimated as follows:

$$
\nu_{e n}=n_{a} \sigma_{e a} V_{\mathrm{th}}^{e},
$$

where $n_{a}$ is the neutral density, $\sigma_{e a}$ is the collision cross section dependent on the electron energy ${ }^{23}\left(\sigma_{e a} \sim(10-40)\right.$ $\times 10^{-20} \mathrm{~m}^{-2}$ for xenon, in the considered energy range), and $V_{\mathrm{th}}^{e}$ is the electron thermal velocity.

However, only including the classical mechanism of collisions cannot explain the electron transport observed in a Hall thruster. This was recognized long ago by many authors. ${ }^{6,7,20,21}$ Until now, however, there is no consensus about which of the possible mechanisms of electron transport is most significant in the Hall thruster. Some suggest that electron collisions with the walls play the major role ${ }^{21}$ while others obtained reasonable solutions including oscillations by assuming only anomalous transport. ${ }^{20}$ Recent experimental data support the idea that the second type of collisions prevails especially near the magnetic field peak. ${ }^{32}$ In the present work we will account for all these possible collisions.

The effective electron collision frequency related to the anomalous transport (Bohm diffusion) can be estimated as

$$
\nu_{\mathrm{B}}=\alpha \omega_{e},
$$




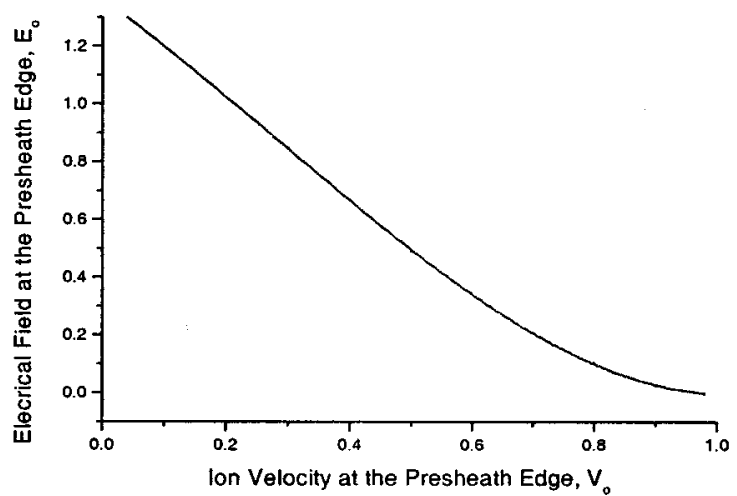

FIG. 3. The relation between the electric field (normalized by $T_{e} / R_{d}$ ) and the ion velocity (normalized by Bohm velocity) at the plasma-sheath interface

where $\alpha \sim 1 / 16$ is the Bohm empirical parameter in the classical formulation. It will be shown in the following that the exact value of this parameter affects the potential drop across the channel. The best fit with the experimental data on the potential drop for a given discharge current corresponds to $\alpha \sim 1 / 44$ instead of the classical value $\sim 1 / 16$. It should be noted that the same conclusion derived by different authors was that the best fit with the experiment corresponds to $\alpha \sim 1 /$ 80-1/100 (Refs. 25 and 33).

Another possible nonclassical mechanism of the electron transport across a magnetic field is due to collisions with a dielectric wall, the so-called near wall conductivity, ${ }^{34,35}$ that accounts for both elastic and nonelastic electron collisions with a wall. According to Ref. 36, the frequency of the electron collisions with a wall can be estimated as

$$
\nu_{e w}=V_{\mathrm{th}}^{e} / h \exp \left(-\Delta \varphi_{w} / T_{e}\right)
$$

where $h=R_{2}-R_{1}$ is the channel width.

\section{Boundary conditions}

In order to obtain a solution of the system of equations (14)-(22) the following boundary conditions must be specified. At the upstream boundary $(z=0)$ we specify the density and velocity similar to Ref. 37 assuming an ion velocity $V_{0}$ $=2 \times 10^{3} \mathrm{~m} / \mathrm{s}$ near the anode that corresponds to an ion temperature of $3 \mathrm{eV}$. This upstream condition implies that we are considering only supersonic plasma flow assuming that the transition from subsonic to supersonic flow ${ }^{25}$ occurs in the anode vicinity. The atom velocity near the anode is assumed to be $V_{0 a}=2 \times 10^{2} \mathrm{~m} / \mathrm{s}$ (Ref. 21 ). The atom density at the anode plane depends upon the mass flow rate that will vary from 2 to $5 \mathrm{mg} / \mathrm{s}$. At the downstream boundary (thruster exit plane, $z=L$ ) we specify an electron temperature of $T_{e}$ $=10 \mathrm{eV}$ (Ref. 21) that is close to that measured in experiment. $^{38}$

\section{Numerical method}

The numerical analysis is similar to that developed previously. ${ }^{39}$ We use the implicit two-layer method to solve the system of equations (14)-(19). These equations are approximated by a two-layer six-point scheme. The electron

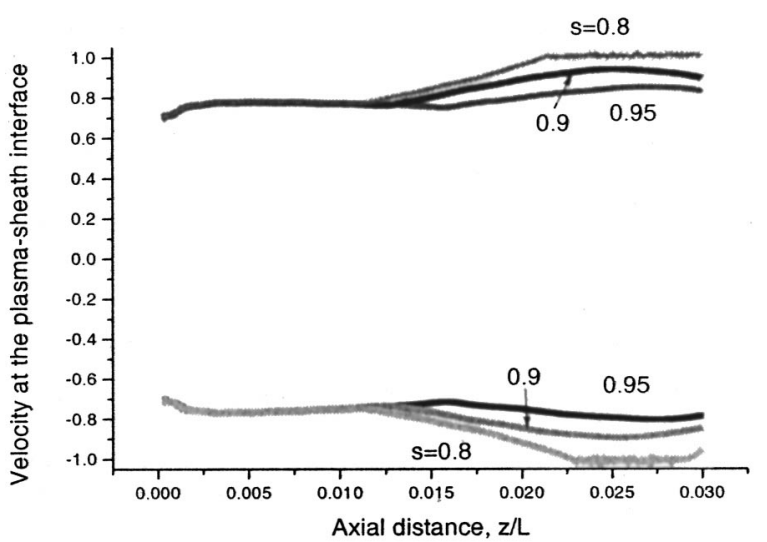

FIG. 4. The radial component of the plasma velocity at the plasma-sheath interface (normalized by the Bohm velocity) along centerline with the secondary electron emission coefficient $s$ as a parameter. The negative velocity corresponds to the inner wall of the channel $\left(r=R_{1}\right)$ and the positive velocity corresponds to the outer wall of the channel $\left(r=R_{2}\right)$

temperature distribution is calculated by iteration initially assuming a trial temperature distribution that satisfies the boundary conditions.

\section{RESULTS}

Computations are performed for the geometry of the SPT-100 (which is a stationary plasma thruster with $100 \mathrm{~mm}$ outer channel wall diameter) that has a channel length of 3 $\mathrm{cm}$, and inner and outer radii are, respectively, 3 and $5 \mathrm{~cm}$ (Refs. 21 and 40). The magnetic field axial profile is similar to that used in Ref. 21 with a magnetic field maximum of $B=160 \mathrm{G}$ near the channel exit plane. All results are presented for the fixed discharge current of $4.5 \mathrm{~A}$ and mass flow rate of $4 \mathrm{mg} / \mathrm{s}$ (xenon). The SEE coefficient will be considered in the range of $0.7-0.95$ that corresponds to electron energy of 15-30 eV in the case of BN (Ref. 41). We have calculated thrust for these conditions which is on the order of $50 \mathrm{mN}$ that is close to that measured in experiment. ${ }^{42}$

First we present an analysis of the sheath solution. As mentioned previously, a monotonic potential distribution in the sheath may be obtained if the Bohm condition is fulfilled at the sheath edge. However if at the same time the electric field at the sheath edge is finite (nonzero), a monotonic solution can be obtained even when the ion velocity at the sheath edge is smaller than that determined by the Bohm condition. The calculated relation between the electric field and the ion velocity at the sheath edge is shown in Fig. 3. One can see that the electric field decreases from the characteristic value of $T_{e} / R_{d}$ ( $R_{d}$ is the Debye length) down to zero when the velocity approaches the Bohm velocity. In the present work, the ion velocity in the quasineutral plasma presheath up to the presheath edge is calculated from Eqs. (14) to (16). This solution therefore establishes the electric field at the sheath-presheath interface.

The plasma density and radial velocity distribution is shown in Fig. 1(b). One can see that the peak density that corresponds to the ionization zone is somewhere in the middle of the channel. The radial velocity component distribution [see Fig. 1(c)] shows that the region where the plasma 


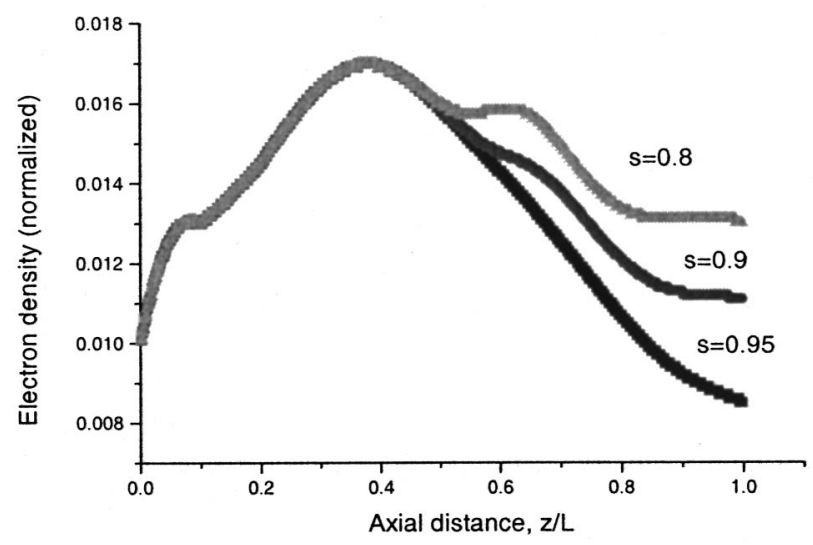

FIG. 5. Plasma density distribution along the thruster channel (centerline) with the secondary electron emission coefficient $s$ as a parameter (normalized by the upstream neutral density).

develops conditions for the entrance to the sheath is close to the lateral walls of the channel. Ions are accelerated in the direction normal to the wall, which is also the direction of the magnetic field lines. This is not a surprising result as the radial electric field is parallel to the magnetic field lines according to Eq. (18). It may be seen also that further downstream the velocity increases at the plasma (presheath) edge. This effect is shown in more detail in Fig. 4 where the velocity at the plasma edge is displayed as a function of axial position. It can be seen that the boundary velocity increases with axial distance from about $0.7 C_{s}$ up to $C_{s}$. The boundary velocity depends also on the secondary electron emission coefficient. Generally speaking, smaller SEE coefficient leads to higher velocity as shown in Fig. 4.

The plasma density distribution along the channel centerline is shown in Fig. 5. Initially the plasma density increases due to ionization and the region where the plasma density has a maximum corresponds to the ionization zone. As result of significant acceleration in the axial direction, the plasma density decreases downstream of the ionization zone as shown in Fig. 5. One can see that near the channel exit plane, the plasma density is higher in the case of the smaller SEE. It will be shown in the following that in this case the

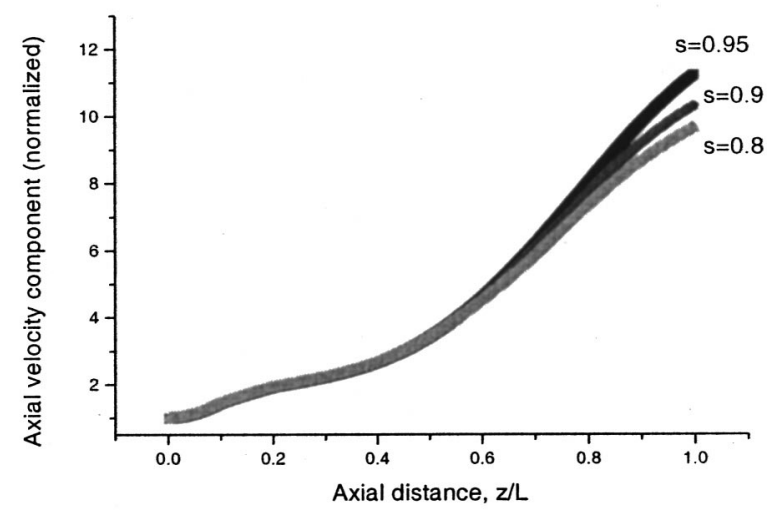

FIG. 6. Neutral density distribution along the channel with the secondary electron emission coefficient $s$ as a parameter (normalized by the upstream neutral density).

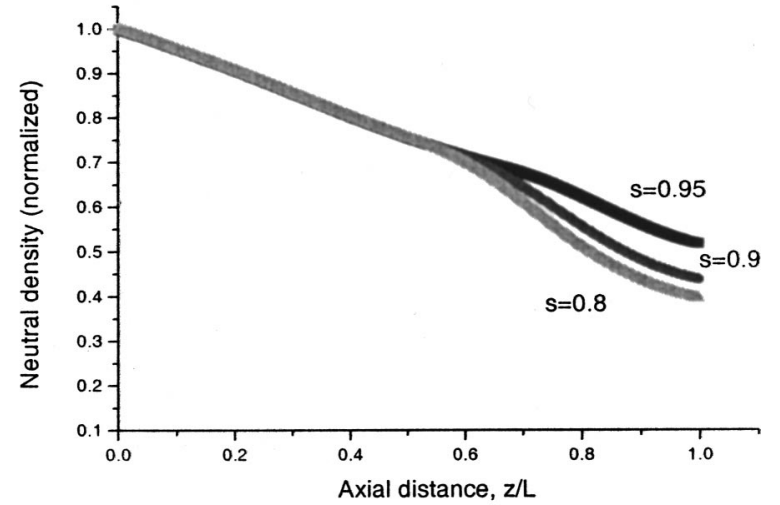

FIG. 7. Axial ion velocity distribution (centerline) along the channel with the secondary electron emission coefficient $s$ as a parameter (normalized by the upstream ion velocity).

electron temperature is high near the channel exit plane that enhances ionization leading to a higher plasma density. As a result of enhanced ionization, the neutral density is smaller in the case of smaller SEE as shown in Fig. 6.

Axial ion velocity distribution along the channel is shown in Fig. 7. It can be seen that the velocity profile is only slightly affected by the SEE. The exit velocity is higher in the case of larger $s$ because in that case the discharge voltage (that accelerates ions) is higher as will be shown in the following.

Axial distribution of the electron temperature is shown in Fig. 8 with SEE coefficient $s$ as a parameter. One can see that the electron temperature peaks at axial distances of about $0.7-0.8 \mathrm{~L}$. The peak electron temperature increases with coefficient $s$ and varies from 15 up to $30 \mathrm{eV}$ when $s$ decreases from 0.95 down to 0.8 . In the first half of the channel, the electron temperature is approximately constant and does not depend on $s$. It should be noted that the electron temperature predicted by the model is in the range that was measured in experiments. ${ }^{32,43,44}$

The current-voltage characteristic of the discharge is shown in Fig. 9 where SEE coefficient $s$ is used as a parameter. It can be seen that the discharge voltage is smaller in the case of the low emissive material for the fixed discharge current. The current-voltage characteristic predicted by the

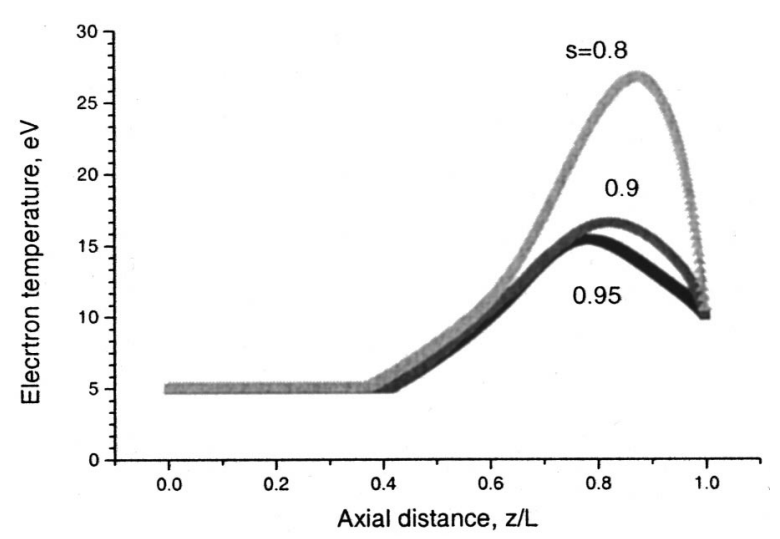

FIG. 8. Electron temperature distribution along the channel with the secondary electron emission coefficient $s$ as a parameter. 


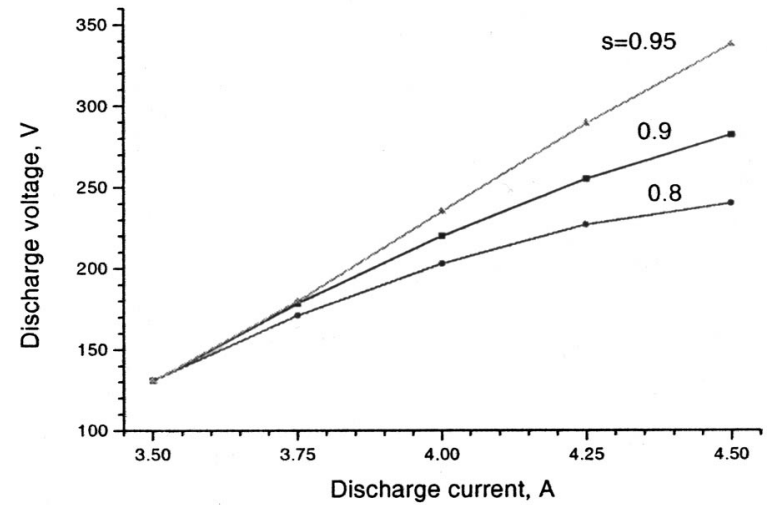

FIG. 9. Current-voltage characteristic of the discharge in cross $E \times B$ field. Effect of the secondary electron emission coefficient.

model and its dependence on the secondary electron emission coefficient are found to be consistent with experiment (Raitses et al. ${ }^{9}$ ). In that experiment, the current-voltage characteristics of two wall materials [namely $\mathrm{BN}$ and glass ceramics (GC)] were measured experimentally and compared. It is expected that the glass ceramics generally has a smaller SEE (note that no data for GC are available, however data on glasses and quartz can be found ${ }^{45}$ ).

\section{DISCUSSION}

This paper presents a traditional two-scale analysis of the plasma-wall interface problem. The approach undertaken is however different from the usual presheath formulation since equations for the presheath are formulated in a two-dimensional manner. Therefore, there are no restrictions on the presheath mechanism (geometric, magnetic, collisions) that usually exist in the one-dimensional formulation. ${ }^{13}$ In fact an important conclusion that comes out from the model is that the presheath has a two-dimensional nature even though the main dependence in the axial direction is in the near wall region. Due to the plasma losses on the wall, the radial density gradient is increased. This density gradient leads to ion acceleration in the radial direction that is the main mechanism that provides the conditions at the sheath edge (presheath mechanism).

Usually a two scale analysis implies that the electric field at the sheath edge equals zero while the velocity equals the Bohm velocity. However, in the specific configuration of the Hall thruster, there may not be a mechanism for ion acceleration up to the Bohm speed. For instance, in the considered conditions, due to the radial expansion and nonelastic collisions, the ion velocity at the quasineutral plasma edge varies from 0.5 to 1 of the Bohm velocity. This means that a monotonic solution for the sheath problem is possible when another additional condition (besides the velocity at the sheath edge) must be formulated. This additional condition was considered previously by several authors. Beilis et al. ${ }^{28}$ and Godyak and Stenberg ${ }^{29}$ proposed the idea of a nonzero electric field of the order of $T_{e} / R_{d}$ at the sheath entrance. The results obtained by Beilis and Keidar ${ }^{19}$ in a magnetic field suggest also that, generally, for the smooth presheathsheath transition, a nonzero electric field at the sheath edge is required. It should be noted that an electric field of the order of $T_{e} / R_{d}$ was also measured recently. ${ }^{46}$ In this paper we show that only a combination of the electric field and the velocity at the sheath edge (with relation between them shown in Fig. 3) can provide smooth plasma-sheath transition along the entire thruster channel. Under the considered conditions, ion acceleration toward the wall varies along the axial distance therefore changing the ion velocity at the sheath edge. In the limiting case, when ions accelerate up to the Bohm speed (near the Hall thruster channel exit plane), the electric field required for the stable solution goes to zero (similar to the traditional two scale problem analysis) as shown in Fig. 3.

It was found that the SEE affects the current-voltage characteristic of the $E \times B$ discharge realized in Hall thrusters. It was shown that the discharge voltage is smaller in the case of low emissive material for a fixed discharge current. The reason for this behavior can be understood as follows. Smaller SEE leads to higher electron temperature since less cold secondary electrons are entering the discharge. As a result, the ionization is enhanced and electron density increases as shown in Fig. 5. Therefore, both ion and electron current density increase that eventually leads to total current increase.

\section{CONCLUSIONS}

The dielectric wall affects the plasma flow inside the Hall thruster channel through the effective coefficient of the secondary electron emission (SEE), $s$. It was found that in the considered condition, the presheath scale length becomes comparable to the channel width so that the plasma channel becomes an effective presheath. The radial ion velocity component at the plasma-sheath interface varies along the thruster channel from about $0.5 C_{s}\left(C_{s}\right.$ is the Bohm velocity) near the anode up to the Bohm velocity near the exit plane, depending on the SEE coefficient. It was obtained that the secondary electron emission significantly affects the electron temperature distribution along the channel. For instance, in the case of $s=0.95$, the electron temperature peaked at about $16 \mathrm{eV}$ while in the case of $s=0.8$ it peaked at about $30 \mathrm{eV}$. The predicted electron temperature is close to that measured experimentally. The model predictions on the dependence of the current-voltage characteristic of the $E \times B$ discharge on the SEE coefficient were found to be consistent with experiment.

\section{ACKNOWLEDGMENTS}

M.K. and I.D.B. gratefully acknowledge financial support by the TRW Foundation.

${ }^{1}$ V. Kim, J. Propul. Power 14, 736 (1998).

${ }^{2}$ R. J. Etherington and M. G. Haines, Phys. Rev. Lett. 14, 1019 (1965).

${ }^{3}$ G. S. Janes and R. S. Lowder, Phys. Fluids 9, 1115 (1966).

${ }^{4}$ A. V. Zharinov and Yu. S. Popov, Sov. Phys. Tech. Phys. 12, 208 (1967).

${ }^{5}$ A. I. Morozov, Sov. Phys. Dokl. 10, 775 (1966).

${ }^{6}$ V. V. Zhurin, H. R. Kaufman, and R. S. Robinson, Plasma Sources Sci. Technol. 8, 1 (1999).

${ }^{7}$ A. I. Morozov and V. V. Savelyev, in Review of Plasma Physics, edited by B. B. Kadomtsev and V. D. Shafranov (Consultants Bureau, New York, 2000), Vol. 21, p. 203. 
${ }^{8}$ E. Choueiri, 37th AIAA Joint Propulsion Conference, Salt Lake City, UT, 2001 (AIAA, Washington DC, 2001), AIAA-2001-3504; Phys. Plasmas 8, 5025 (2001)

${ }^{9}$ Y. Raitses, J. Ashkenazy, G. Appelbaum, and M. Guelman, 25th International Conference on Electric Propulsion, Cleveland, $\mathrm{OH}$ (The Electric Rocket Propulsion Society, Worthington, OH, 1997), IEPC 97-056.

${ }^{10}$ Y. Raitses, L. Dorf, A. Litvak, and N. Fisch, J. Appl. Phys. 88, 1263 (2000).

${ }^{11}$ Y. Raitses, D. Staack, A. Smirnov, A. A. Litvak, L. A. Dorf, T. Graves, and N. J. Fisch, in Ref. 8, AIAA-2001-3776.

${ }^{12} \mathrm{D}$. Bohm, in The Characteristics of Electrical Discharges in Magnetic Field, edited by A. Guthry and R. K. Wakerling (McGraw-Hill, New York, 1949).

${ }^{13}$ K. U. Riemann, J. Phys. D 24, 493 (1991).

${ }^{14}$ R. Chodura, Phys. Fluids 25, 1628 (1982).

${ }^{15}$ K. U. Riemann, Phys. Plasmas 1, 552 (1994).

${ }^{16}$ H. Schmitz, K. U. Reimann, and Th. Daube, Phys. Plasmas 3, 2486 (1996).

${ }^{17}$ M. Keidar, I. Beilis, R. L. Boxman, and S. Goldsmith Proceeding XXII ICPIG, Stevens Institute of Technology, Hoboken, NJ, 1995, Vol. 2 p. 157.

${ }^{18}$ I. I. Beilis, M. Keidar, and S. Goldsmith, Phys. Plasmas 4, 3461 (1997).

${ }^{19}$ I. I. Beilis and M. Keidar, Phys. Plasmas 5, 1545 (1998).

${ }^{20} \mathrm{~J}$. M. Fife and M. Martinez-Sanchez, 24th International Conference on Electric Propulsion, Moscow, Russia, 1995 (The Electric Rocket Propulsion Society, Worthington, OH, 1995), IEPC-95-240.

${ }^{21}$ J. P. Bouef and L. Garrigues, J. Appl. Phys. 84, 3541 (1998).

${ }^{22}$ I. D. Boyd, L. Garrigues, J. Koo, and M. Keidar, 36th AIAA Joint Propulsion Conference, Huntsville, AL, 2000 (AIAA, Washington DC, 2000), AIAA-2000-3520.

${ }^{23}$ I. Katz and M. Mandell, Bull. Am. Phys. Soc. 45, 165 (2000).

${ }^{24}$ A. Fruchtman and N. J. Fisch, 34th AIAA Joint Propulsion Conference, Cleveland, OH, 1998 (AIAA, Washington DC, 1998), AIAA-1998-3500.

${ }^{25}$ E. Ahedo, P. Martinez, and M. Martines-Sanches, in Ref. 22, AIAA-20003655.

${ }^{26}$ K. Makowsky, Z. Peradzynski, N. Gascon, and M. Dudeck, 35th AIAA Joint Propulsion Conference, Los Angeles, CA, 1999 (AIAA, Washington DC, 1999), AIAA-99-2295.
${ }^{27}$ S. Locke, U. Schumlak, and J. M. Fife, in Ref. 8, AIAA-01-3327.

${ }^{28}$ I. I. Beilis, V. A. Bityurin, U. A. Vasiljeva, V. V. Kirillow, G. A. Lyubimov, S. A. Medin, A. E. Sheindlin, and B. Ya. Shumjatsky, MHD Energy Conversion. Physical and Technical Aspects, edited by V. A. Kirillin and A. E. Sheyndlin (Nauka, Moscow, 1983), 368 pp. English translation: (AIAA, New York, 1986).

${ }^{29}$ V. A. Godyak and N. Sternberg, IEEE Trans. Plasma Sci. 18, 159 (1990).

${ }^{30}$ G. D. Hobbs and J. A. Wesson, Plasma Phys. 9, 85 (1967).

${ }^{31}$ A. I. Morozov and V. V. Savel'ev, in Ref. 20, IEPC-95-161.

${ }^{32}$ N. B. Meezan, W. A. Hargus, and M. A. Cappelli, Phys. Rev. E 63, 026410 (2001).

${ }^{33}$ J. M. Fife and S. Locke, AIAA Aerospace Sciences Meeting and Exhibit, Reno, NV, January 2001 (AIAA, Washington DC, 2001), AIAA-20011137.

${ }^{34}$ A. I. Morozov, Prikl. Mekh. Tekh. Fiz. 3, 19 (1968).

${ }^{35}$ A. I. Morozov and V. V. Savel'ev, Plasma Phys. Rep. 27, 570 (2001).

${ }^{36}$ V. I. Baranov, Yu. S. Nazarenko, V. A. Petrosov, A. I. Vasin, and Yu. M. Yashnov, in Ref. 9, IEPC-97-060.

${ }^{37}$ A. I. Morozov and V. V. Savel'ev, Plasma Phys. Rep. 26, 219 (2000).

${ }^{38}$ S. Kim, J. E. Foster, and A. D. Gallimore, 32nd AIAA Joint Propulsion Conference, Orlando, FL (AIAA, Washington DC, 1996), AIAA-96-2972, July 1996.

${ }^{39}$ M. Keidar, I. Beilis, R L. Boxman, and S. Goldsmith, J. Phys. D 29, 1973 (1996).

${ }^{40}$ L. B. King, A. D. Gallimore, and C. M. Marrese, J. Propul. Power 14, 327 (1998).

${ }^{41}$ J. P. Bugeat and C. Koppel, in Ref. 20, IEPC-95-35.

${ }^{42}$ S. Bechu, G. Perot, N. Gascon, P. Lasgorceix, A. Hauser, and M. Dudeck, in Ref. 26, AIAA-99-2567.

${ }^{43}$ A. M. Bishaev and V. Kim, Sov. Phys. Tech. Phys. 23, 1055 (1978).

${ }^{44}$ J. M. Haas and A. D. Gallimore, in Ref. 22, AIAA-00-3422.

${ }^{45}$ Handbook of Chemistry and Physics, edited by R. C. Weast (The Chemical Rubber Co., Boca Raton, FL, 1965), p. E-138.

${ }^{46} \mathrm{H}$. A. Kamal and N. Hershkowitz, 25th International Conference on Plasma Science, Raleigh, NC, 1998 (IEEE Nuclear and Plasma Science Society, Conference Record-Abstracts, IEEE Catalog No. 98CH36221), p. 166. 